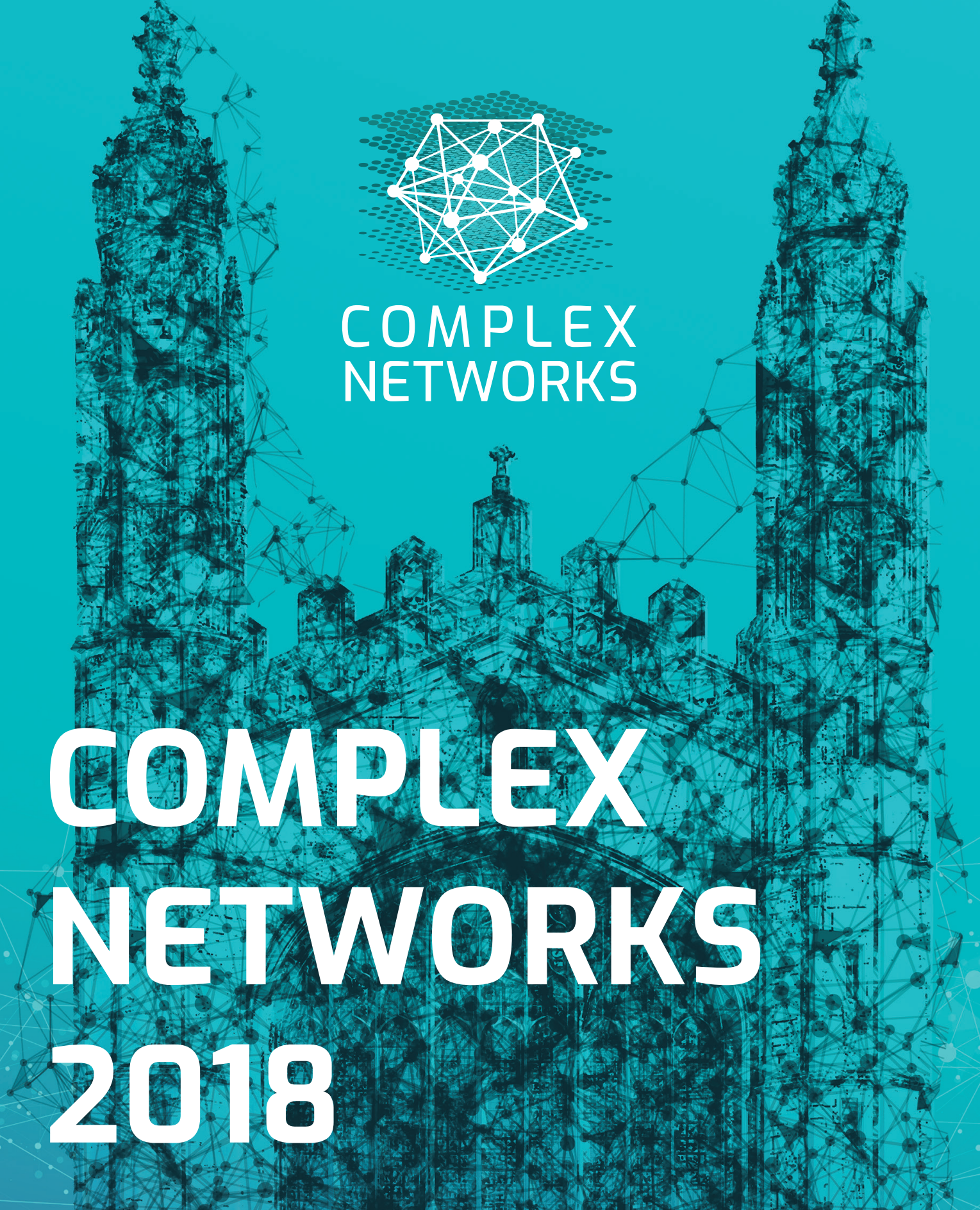

THE I I INTERNATIONAL CONFERENGE
ON COMPLEX NET WORKS AND THEIR APPLICATIONS

December 11 - 13, 2018 Cambridge United Kingdom

Book of Abstracts 


\section{COMPLEX NETWORKS 2018}

The $7^{\text {th }}$ International Conference on Complex Networks \& Their Applications

December 11 - 13, 2018 Cambridge, United Kingdom

Published by the International Conference on Complex Networks \& Their Applications.

Editors:

Hocine Cherifi

University of Burgundy, France

Pietro Lio'

University of Cambridge, UK
Luca Maria Aiello

Nokia-Bell Labs, UK

Renaud Lambiotte

Oxford University, UK
Chantal Cherifi

University of Lyon, France

Luis Mateus Rocha Indiana

University, USA

COMPLEX NETWORKS 2018

e-mail: hocine.cherifi@u-bourgogne.fr

Copyright Notice COMPLEX NETWORKS 2018 and the Authors

This publication contributes to the Open Access movement by offering free access to its articles and permitting any users to read, download, distribute, print, search, or link to the full texts of these articles, crawl them for indexing, pass them as data to software. The copyright is shared by authors and the $7^{\text {th }}$ International Conference on Complex networks \& Their Applications (COMPLEX NETWORKS 2018) to control over the integrity of their work and the right to be properly acknowledged and cited.

To view a copy of this license, visit http://www.creativecommons.org/licenses/by/4.0/

The use of general descriptive names, registered names, trademarks, service marks, etc. in this publication does not imply, even in the absence of a specific statement, that such names are exempt from the relevant protective laws and regulations and therefore free for general use. While the advice and information in this book are believed to be true and accurate at the date of publication, neither the authors nor the editors nor the publisher can accept any legal responsibility for any errors or omissions that may be made. The publisher makes no warranty, express or implied, with respect to the material contained her.

ISBN: 978-2-9557050-2-5 


\title{
Extracting Multi-Layer Networks from Sentinel-2 Satellite Image Time Series
}

\author{
Roberto Interdonato, Raffaele Gaetano, Danny Lo Seen, and Mathieu Roche \\ CIRAD - UMR TETIS, Montpellier, France \\ \{roberto.interdonato, raffaele.gaetano, danny.lo_seen, mathieu.roche\}@cirad.fr
}

\section{Introduction}

Nowadays, modern Earth Observation systems continuously generate huge amounts of data. The Sentinel-2 (S2) Earth Observation mission, developed by the European Space Agency (ESA) as part of the Copernicus Programme, supplies image acquisition at high spatial resolution (up to $10 \mathrm{~m}$ ) with high temporal revisit period (every 5 days). This information can intuitively be organized into time series of high-resolution satellite imagery, used for area monitoring tasks. Moreover, since S2 SITS are publicly available, these type of data is also attractive for research purposes.

The successful application of classification approaches based on deep learning to remote sensing tasks [5] led to significant improvements in the development of land cover maps, i.e., allowing to automatically learn complex feature representations from satellite images. Nevertheless, different representation models for satellite images can be defined, i.e., based on complex network models, which may allow to discover new relations between the spatial objects in an image and to apply existing analysis techniques to a new domain.

The use of network analysis techniques in the remote sensing domain has often been neglected. Our hypothesis is that a network model would lead to a spatial representation of satellite data that goes beyond conventional mapping, taking into account in particular the different types of relationships between objects or segments identified in the images. The aim of this work is to show how Satellite Image Time Series (SITS) can be profitably represented using complex network models, by proposing a method to extract a Multilayer Network from a SITS.

This will enable the application of network analysis techniques to remote sensing data, paving the way for the definition of innovative approaches able to take into account, together with the spectral and textural information, the contextual information deriving from the spatial organization of the entities in a landscape. As next step, we plan to use multilayer community detection approaches to investigate how the spatial organization of the landscape suggested by the community structure evolves over time, i.e., identifying communities which correspond to sets of image segments or specific objects. While global approaches [4] will help studying the organization of the whole area depicted in the SITS, local ones [2] will allow to focus on specific objects or zones of interest. Their Applications. 11 - 13 Dec., 2018, Cambridge (UK) 


\section{Proposed Model}

In this work, we will propose a methodology to build a multilayer network [3] starting from satellite image time series. In our case, we intend to represent in each layer the image coming from a different timestamp in the series, i.e., obtaining an ordered multilayer network modeling the evolution of a spatial area in a certain time range. Given a SITS containing a set $S=\left\{s_{0}, \ldots, s_{n}\right\}$ of $n$ images representing the same scene at $n$ different timestamps, the aim is to obtain a multilayer network $G=(V, E, L)$, where $V$ is a set of nodes, $L$ is a set of layers of size $n$ (one for each image/timestamp), and $E \subseteq(V \times L) \times(V \times L)$ is a set of edges (i.e., connecting node-layer couples). We will now describe in detail the three main steps of the proposed methodology: Image segmentation, Interlayer edges insertion and Intralayer edges insertion.

Image segmentation. At the first step of our procedure, each image $s_{i} \in S$ is partitioned in $k_{i}$ segments, i.e., coherent sets of pixels. Each segment of image $s_{i}$ will then correspond to a node in layer $l_{i}$; note that the segmentation of each image is performed independently, i.e., the number of nodes in each layer may be different. In our example, for efficiency and scalability reasons, we used Felsenszwalbs image segmentation [1] in its Scikit-Image python implementation ${ }^{1}$, but any other image segmentation algorithm may be used in its place.

Interlayer edges insertion.. The second step includes the insertion of the edges connecting nodes in different layers. In other cases (e.g., multiplex networks) only coupling interlayer edges may be allowed, i.e., connecting nodes which represent different instances of the same entity. Conversely, in our case it is not possible to have a 1:1 correspondence between nodes in different layers, due to the independent segmentation processes. For this reason, we insert an interlayer edge $\left(v_{i}, l_{a}, v_{j}, l_{b}\right)$ s.t. $a \neq b$ if the segments corresponding to nodes $v_{i}$ and $v_{j}$ have at list a common pixel (i.e., there is an overlap between the segments). Due to the temporal nature of the network, interlayer edges are only inserted between a layer and the subsequent one (i.e., $b=a+1$ ), following the time series ordering.

Intralayer edges insertion. The last step consists in adding the edges between the nodes in each layer. For this step we resort to Region Adjacency Graphs (RAGs) ${ }^{2}$, i.e., an edge $\left(v_{i}, l_{a}, v_{j}, l_{a}\right)$ between two nodes in the same layer exists if the segments corresponding to nodes $v_{i}$ and $v_{j}$ are adjacent. Edges are weighted based on the difference of average RGB color between the two segments, i.e., lower weights will correspond to most similar segments. In practical cases, simple refinement procedures may be applied, e.g., merging similar nodes (i.e., the ones connected by edges showing lower weights) or disconnecting dissimilar ones (i.e., the ones connected by edges showing higher weights).

\section{Results}

For this example, we consider a SITS of High Spatial Resolution Sentinel-2 images of $100 \mathrm{~km} \times 100 \mathrm{~km}$, depicting the Koumbia area (Tuy Province in Burkina Faso). The SITS

\footnotetext{
${ }^{1}$ http://scikit-image.org/docs/dev/api/skimage.segmentation.html

${ }^{2} \mathrm{http} / / /$ scikit-image.org/docs/dev/auto_examples/segmentation/plot_rag_draw.html
} 
consists of 23 images referring to the period 2016/01/16-2016/12/21. Table 1 reports main structural characteristics of the multilayer network obtained with the proposed method, while Figure 1 shows the distribution of nodes, intralayer and interlayer edges over the 23 layers. The number of nodes in each layer is rather stable, except for a lower peak at layer 18, maybe due to an excessive amounts of clouds in the image. Given the adjacency criterion used in the RAGs construction, the number of intralayer edges is clearly proportional to the number of nodes (the variability among the layers here is naturally emphasized). The number of interlayer edges (intended as number of interlayer edges outgoing from each layer) is slightly more unstable. Even if some proportionality with the number of nodes can still be recognized, this characteristic is also biased by the outcome of the segmentation process (which in turn depends from the landscape changes during time). Nevertheless, given the complexity of the process and the nature of the input data, an average interlayer degree of 3.99 is fairly reasonable, considering that it represents the average number of segments at time/layer $t+1$ which overlap with a segment taken at time/layer $t$.

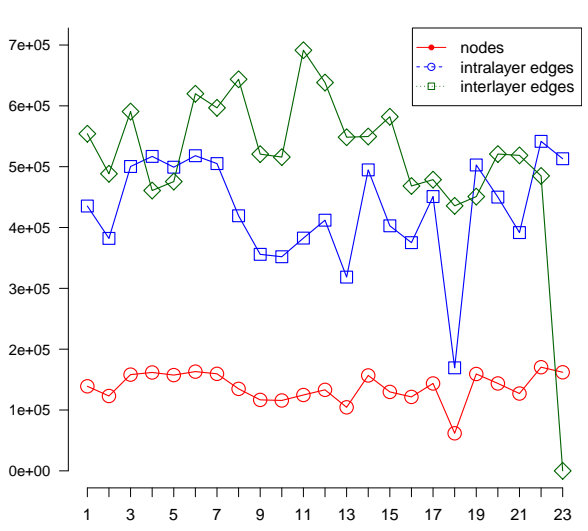

Fig. 1. Distribution of nodes, intralayer and interlayer edges over the 23 layers.

\begin{tabular}{|c|c|}
\hline layers & 23 \\
\hline tot nodes & 3167802 \\
avg nodes & 137730 \\
max nodes & 170398 \\
\hline \hline tot intra edges & 9889167 \\
avg intra edges & 429963 \\
max intra edges & 541649 \\
\hline tot inter edges & 11833726 \\
avg inter edges & 514509 \\
max inter edges & 691431 \\
\hline \hline Density & $4.4 e-05$ \\
Adeg (intra) & 116 \\
Adeg (inter) & 3.99 \\
\hline
\end{tabular}

Table 1. Structural characteristics of the multilayer network.

\section{References}

1. Pedro F. Felzenszwalb and Daniel P. Huttenlocher. Efficient graph-based image segmentation. International Journal of Computer Vision, 59(2):167-181, 2004.

2. R. Interdonato, A. Tagarelli, D. Ienco, A. Sallaberry, and P. Poncelet. Local Community Detection in Multilayer Networks. Data Mining and Knowledge Discovery, Jul 2017.

3. M. Kivela, A. Arenas, M. Barthelemy, J. P. Gleeson, Y. Moreno, and M. A. Porter. Mutilayer networks. Journal of Complex Networks, 2(3):203-271, 2014.

4. P. J. Mucha, T. Richardson, K. Macon, M. A. Porter, and J.-P. Onnela. Science. Community structure in time-dependent, multiscale, and multiplex networks, 328(5980):876-878, 2010.

5. X. X. Zhu, D. Tuia, L. Mou, G. Xia, L. Zhang, F. Xu, and F. Fraundorfer. Deep learning in remote sensing: A comprehensive review and list of resources. IEEE Geoscience and Remote Sensing Magazine, 5(4):8-36, 2017. 